\title{
ANÁLISIS DEMOGRÁFICO DE LA RAZA EQUINA HISPANO-ÁRABE
}

\author{
DEMOGRAPHIC ANALYSIS OF THE EQUINE BREED SPANISH-ARABIC
}

\author{
Gómez, M. ${ }^{1 *}$, León, J.M. ${ }^{1}$ y Delgado, J.V. ${ }^{1}$ \\ ${ }^{1}$ Departamento de Genética. Universidad de Córdoba. Edificio Gregor Mendel. Córdoba. España. \\ *z72gocam@uco.es
}

\section{PALABRAS CLAVE ADICIONALES}

Caballos. Tamaño efectivo. Intervalo generacional.

\section{RESUMEN}

El esquema de selección del caballo HispanoÁrabe se encuentra en desarrollo por lo que es necesario conocer su estructura poblacional a partir de la información registrada en el Libro Genealógico para diseñar una estrategia de mejora y utilización.

Se calcularon los parámetros demográficos, sobre un censo total de 1041 animales que forman parte del núcleo selectivo de la raza. El tamaño efectivo obtenido fue de 1036,94 animales (553 hembras y 488 machos), con un ratio sexual de 1,13. Si el manejo reproductivo es el correcto, habría un incremento de la endogamia por generación de $0,000482(0,048 \%)$ y por año, de $0,000055(0,005 \%)$, algo esperado debido al intervalo generacional total de 8,65 años (hembras 8,95 y machos 8,31 ). El valor obtenido fue alto pero común en la especie equina, donde muchos animales se utilizan sólo para carreras deportivas. La tasa de reposición observada en hembras fue de $12,66 \%$ y en machos, de $11,68 \%$, con una edad media para machos y hembras de 8 y 9 años, respectivamente. Se deduce que el progreso genético se está gestionando de manera adecuada, manteniendo la variabilidad genética y dinámica de la raza.

\section{SUMMARY}

The breeding program of the Spanish-Arabian horse is in process of development and, for this reason, is necessary to know its population structure from the information registered in the Herd Book to design strategy for its improvement and utilization.

Presentado al Congreso SERGA (2010, Asturias).

\section{Additional KeYWORDS}

Horses. Effective size. Generation interval.

The demographic parameters were calculated having a census of 1041 animals, taking part of the selective nucleus; the effective population size was 1036.94 (553 females and 488 males) with a sex ratio of 1.13 .

With a correct reproductive management we expect an increasing of the inbreeding rate by generation of $0.000482(0.048 \%)$ and by year of $0.000055(0.005 \%)$, something expected because the total generational interval of 8.65 years (females 8.95 and males 8.31), value too high in the equine species, but common, because of a lot of animals are employed only in sport activities. The level of replacement observed was $12.66 \%$ in females and $11.68 \%$ in males, with mean ages of 8 and 9 years in males and females respectively. The good management of the genetic progress is demonstrated, also maintaining the level of genetic variability and dynamism of the breed.

\section{INTRODUCCIÓN}

La raza equina Hispano-árabe, es originaria de Andalucía, actualmente esta región es un principal área de distribución pero se encuentra distribuida por todo el territorio peninsular. La Unión Española de Ganaderos de Pura Raza Hispano-Árabe (UEGHá) es la gestora del Libro Genealógico de la raza; la cual, está clasificada en peligro de extinción, según el Catálogo oficial de razas de Ganado de España (BOE, 2009). Las razas autóctonas constituyen parte del patrimonio histórico y cultural, y su salvaguarda es una prioridad indiscutible (León, 2008). Así pues, la adecuada gestión de la diversidad 
genética es fundamental para su sustentabilidad (UNEP, 1992), siendo imprescindible su caracterización demográfica a la hora de afrontar el diseño de una estrategia correcta para su utilización. Según Gama (2002), la caracterización de un sistema de producción, incluyendo el profundo conocimiento de la estructura demográfica, debe constituirse en la primera etapa de cualquier programa de mejora genética. Conocer la estructura de una población, su variabilidad y flujo de genes, es necesarios antes de empezar un programa de selección; el análisis demográfico pone de relieve las circunstancias que afectan a la población (Valera et al., 2005).

El estudio de la constitución genética de las poblaciones y su evolución a través de las generaciones, es la base para el establecimiento y desarrollo de programas de selección o de conservación (Domínguez et al., 2010). Desde la aprobación del esquema oficial de la raza Hispano-Árabe se realiza el estudio demográfico incluyendo los censos, los intervalos generacionales, el tamaño efectivo, los ratios sexuales, incrementos de la endogamia por generación y por año, tasas de reposición, distribución por sexos y edad media.

\section{MATERIAL Y MÉTODOS}

Se utilizó la base de datos registrada en el programa de gestión del Libro Genealógico de la raza, compuesto de un archivo de animales nacidos entre 1987 y 2009, con genealogías contrastadas. El archivo definitivo sobre el cual se trabajó, fue en formato EXCEL y se organizaron los animales por edades y por sexos. Los parámetros demográficos fueron calculados con diversas aplicaciones informáticas; en primer lugar, los censos totales (sexo y edad), utilizando el procedimiento PROC MEANS y PROC FREC del paquete estadístico SAS en su versión 9.0. Siguiendo las recomendaciones de Kinghorn (2000), se obtuvo las pirámides de edades y el cálculo de los interva- los generacionales totales y por sexos. Con esta misma información, se obtuvo la distribución de la población por sexos y, posteriormente, la determinación del ratio sexual. Se procedió al cálculo de la tasa de reposición total $\left(\mathrm{T}_{\mathrm{t}}=\mathrm{N}_{1} / \mathrm{N}_{\mathrm{t}}\right)$, tanto en machos $\left(\mathrm{T}_{\mathrm{m}}=\right.$ $\left.\mathrm{N}_{1} / \mathrm{N}_{\mathrm{mt}}\right)$ como en hembras $\left(\mathrm{T}_{\mathrm{h}}=\mathrm{N}_{\mathrm{l}} / \mathrm{N}_{\mathrm{ht}}\right)$. Para poblaciones con diferente número de machos y hembras, el tamaño efectivo de la población se calculó de acuerdo con lo propuesto por Falconer y Mackay (1996). Finalmente se calculó el incremento de la consanguinidad por año $(\Delta \mathrm{F} / \mathrm{año})$. A partir de ésta se determina el incremento de consanguinidad por generación $(\Delta \mathrm{F} /$ generación), como ( $\Delta \mathrm{F} / \mathrm{año} \mathrm{x} \mathrm{L})$, dónde L representa el intervalo generacional total (Wright, 1992; Stone, 1977).

\section{RESULTADOSYDISCUSIÓN}

La eficacia de la metodología empleada en el presente trabajo ha sido contrastada en poblaciones de animales domésticos que se encuentran en la frontera entre la conservación y la mejora genética, como es el caso del caballo Lusitano en la especie equina (Vicente et al., 2009) o bien la experiencia en la raza canina Fila de San Miguel (Cruz y Costa, 2009).

En la actualidad el núcleo selectivo de la raza es de 1041 animales, que es un número suficiente para empezar con el programa de mejora de la raza equina Hispano-Árabe, distribuidos por sexos de 553 hembras y 488 machos, resultados que

Tabla I. Distribución de censos de la población. (Distribution of the population census).

\begin{tabular}{cccc}
\hline Edad (años) & Hembras & Machos & Total \\
\hline $1-5$ & 262 & 225 & 487 \\
$6-10$ & 114 & 103 & 217 \\
$11-15$ & 76 & 86 & 162 \\
$16-20$ & 61 & 57 & 118 \\
$>20$ & 40 & 17 & 57 \\
Total & 553 & 488 & 1041 \\
\hline
\end{tabular}




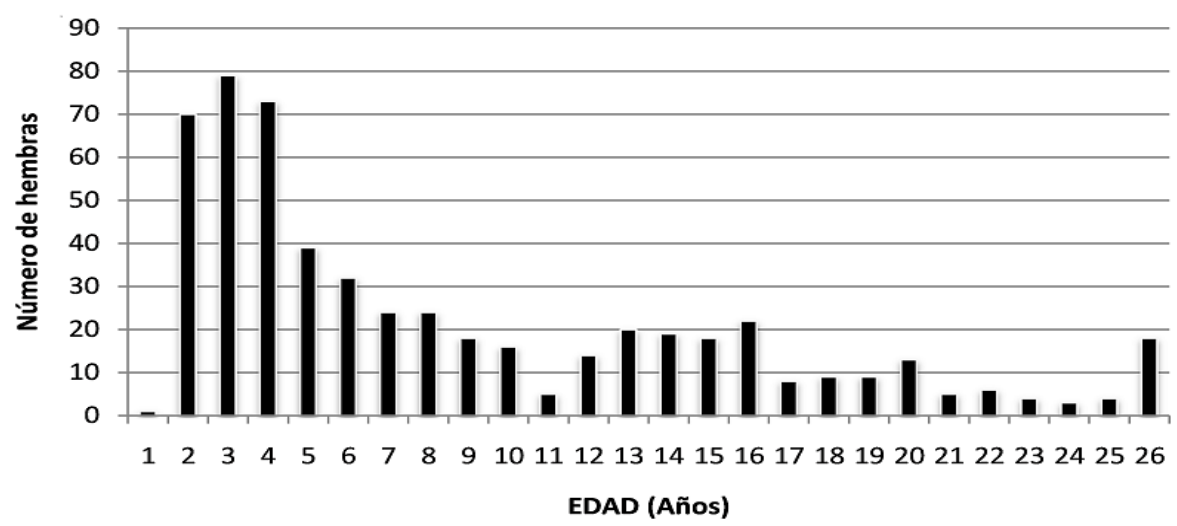

Figura 1. Pirámide de edades en hembras de la población Hispano-Árabe.(Females age pyramid of the Hispano-Arab population).

se observan en la tabla I.

En las pirámides de edades por sexos, observamos en las hembras (figura 1), una mayor agrupación de animales entre 2-8 años, y en los machos (figura 2) se aprecia una mayor concentración entre 2-7 años. El ratio sexual encontrado, de $1 / 1,13$, es una cifra muy baja en caballos si lo comparamos con otras razas que presentan una proporción $1 / 5$. Este resultado demuestra la intensa expansión de la raza con la utilización de la inseminación artificial, hecho que influye en la reducción significativa del ratio sexual.
En cuanto a la tasa de reposición en machos y hembras $(11,68 \%$ y $12,66 \%$ respectivamente), se encontraron valores normales dentro de la especie algo que contrasta con lo comentado anteriormente.

En la especie equina, generalmente por su longevidad, la vida reproductiva empieza a los 4 años de edad. Van der Werf(2000), expuso que el intervalo generacional es la edad media de los progenitores cuando su primera descendencia ha nacido. Para el caso de las hembras y machos se obtuvo un intervalo de 8,95 y 8,31 respectivamente.

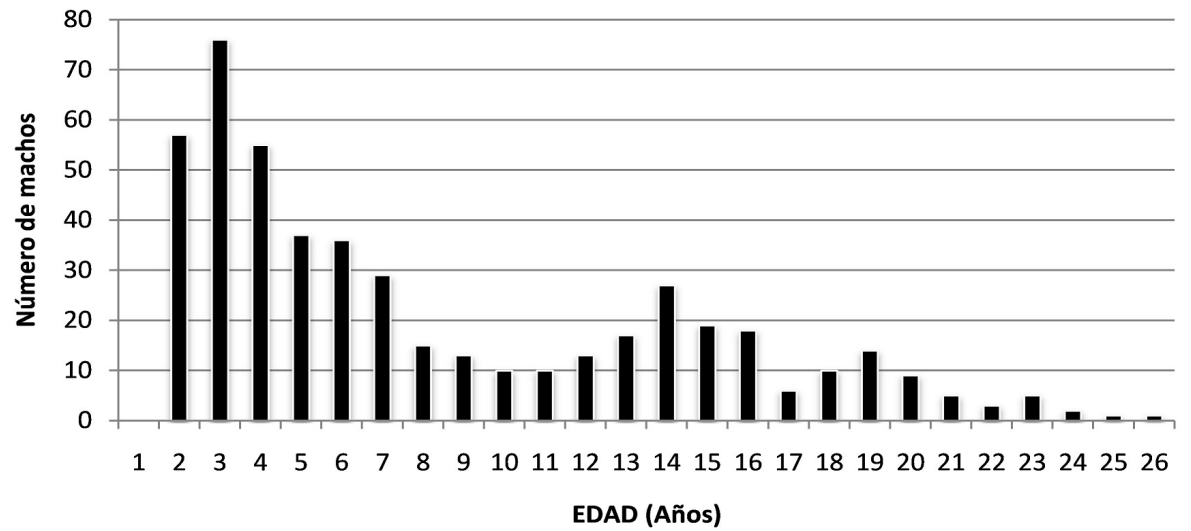

Figura 2. Pirámide de edades de los machos de la población Hispano-Árabe. (Age pyramid of male Hispanic-Arab population). 


\section{GÓMEZ, LEÓNY DELGADO}

Por otra parte, si el manejo reproductivo es el correcto tendríamos un incremento de la endogamia por generación de 0,000482 $(0,048 \%)$ y por año de $0,000055(0,005 \%)$, algo esperado debido al intervalo generacional, de 8,65 años, resultado relativamente bajo si lo contrastamos con los obtenidos en el Pura Raza Español (PRE) de 10,11 años (Valera et al., 2005).

El tamaño efectivo $\left(\mathrm{N}_{\mathrm{e}}\right)$ fue de 1036,94 animales, de acuerdo con lo propuesto por Falconer y Mackay (1996). Este número de individuos sería la población real para mantener las tasas de consanguinidad, si tuvieran la condición ideal, desde el punto de vista reproductivo. El $\mathrm{N}_{\mathrm{e}}$ y la tasa de consanguinidad son los parámetros más importantes en la conservación de las pequeñas poblaciones de animales domésticos. Estos dos parámetros son referencia en el mundo

\section{BIBLIOGRAFÍA}

BOE. 2009. Real Decreto 2129/2008, de 26 de diciembre, por el que se establece el Programa nacional de conservación, mejora y fomento de las razas ganaderas. Secc. I. pp. 9211-9242.

Falconer, D.S. and Mackay, T.F.C. 1996. Introduction to quantitative genetics. $4^{\text {th }}$ ed. Longman. Essex. England.

Cruz, C. e Costa, J.S. 2009. Aspectos demográficos e avaliação do estatuto de risco da raça cão de Fila de São Miguel. Arch. Zootec., 58: 505-508.

Domínguez, J., Rodríguez, F.A., Núñez, R., Ramírez, R., Ortega, J.A. y Ruíz, A. 2010. Análisis del pedigrí y efectos de la consanguinidad en el comportamiento del ganado de Lidia Mexicano. Arch. Zootec., 59: 63-72.

Gama, L.T. 2002. Melhoramiento genético animal. Escolar Editora. Lisboa. Portugal.

Kinghorn, B.P. 2000. The genetic basis of crossbreeding. In: Kinghorn, B.P., Van der Werf, J.H.J. and Ryan, M. (Eds.). Animal breeding. Use of new technologies. Chapter 4. The Post Graduate Foundation in Veterinarian Science. University of Sydney.

León, J.M. 2008. Evaluación del esquema de selección de la raza caprina Murciano-Granadina. de la conservación, hasta el punto que sirven de referencia en organismos nacionales e internacionales para decidir qué poblaciones se encuentran en peligro de extinción, en desarrollo o deben ser consideradas razas de fomento.

Los resultados del presente estudio demográfico del caballo Hispano-Árabe permiten resaltar algunos parámetros importantes, para su conservación y mejora, del tamaño efectivo de la población, se desprende que no hay peligro de extinción y si clara vocación de expansión. El incremento de la consanguinidad por generación y por año, sugiere que no hay peligro de perder variabilidad genética. De los demás parámetros demográficos, se deduce gestión adecuada tanto por la dirección técnica del esquema, como por la UEGHá encargada de la conservación y mejora de la raza.

Tesis Doctoral. Universidad de Córdoba.

Stone, B. 1977. Cálculo de los coeficientes de consanguinidad. Agric. Rec., 3: 56-58.

UNEP. 1992. Rio declaration. World Conference on Environment and Development. United Nations Environment Program. Brazil. United Nations Environment Program. United Nations publication. Sales No. E.73.II.A.14 and corrigendum. Río de Janeiro. Brasil.

Valera, M., Molina, A., Gutiérrez, J.P. and Gómez, Goyache, J. 2005. Pedigree analysis in the Andalusian horse: population structure, genetic variability and influence of the Carthusian strain. Livest. Prod. Sci., 95: 57-66.

Van der Werf, J.H.J. 2000. Livestock straight breeding system structures for the sustainable intensification of extensive grazing systems. In: S. Galal, J. Boyazoglu e K. Hammond (Eds.). Workshop on developing breeding strategies for lower input animal production environments. ICAR Technical Series, 3: 105-178.

Wright S. 1922. Coefficients of inbreeding and relationship. Am. Nat., 56: 330-338.

Vicente, A., Carolino, N. e Gama, L.T. 2009. Indicadores demográficos no cavalo Lusitano. Arch. Zootec., 58: 501-504. 